\title{
Laser intensity profile based terahertz field enhancement from a mixture of nano-particles embedded in a gas
}

\author{
P. Varshney ${ }^{1}$, A. P. Singh ${ }^{2}$, M. Kundu ${ }^{1,3}$, and K. Gopal ${ }^{2, *}$ \\ ${ }^{1}$ Institute for Plasma Research, HBNI, Bhat Gandhinagar-382428, Gujarat -India \\ ${ }^{2}$ Department of Physics and Electronics, Rajdhani College (University of Delhi), Delhi-110015, India \\ ${ }^{3}$ Homi Bhabha National Institute, Training School Complex, Anushakti nagar, Mumbai 400094, India \\ "Email: kgopal874u@gmail.com
}

\begin{abstract}
Nano-particle embedded system plays an importance in developing of future terahertz $(\mathrm{THz})$ radiation source for real-world applications. The laser interactions with nanoparticle embedded system can produce a wide range of $\mathrm{THz}$ radiation due to plasma oscillations excitation. We investigate $\mathrm{THz}$ field generation from the laser-beat wave interaction with a mixture of spherical and cylindrical graphite nanoparticles (NPs) in argon gas. Different laser intensity distributions such as Gaussian, cosh-Gaussian, flat-top and ring shape laser pulses have been studied in this work. The relevant plasmon resonance conditions with appropriate symmetry of spherical nanoparticles (SNPs) and cylindrical nanoparticles (CNPs) are discussed. THz field is enhanced upto the order of $10^{2}$ when the laser intensity redistributes along the polarization direction for a ring shape field envelope.
\end{abstract}

Keywords: Surface plasmon resonance, nano-particles and terahertz radiation 


\section{Introduction}

Terahertz $(\mathrm{THz})$ radiation lies in between microwave and infrared range of electromagnetic (EM) wave spectrum. It has a number of applications in various areas e.g. medical imaging, terahertz spectroscopy, materials characterization and telecommunications. Conventional sources for $\mathrm{THz}$ radiation e.g. optical rectification(Faure et al., 2004), photoconductive antenna (Jepsen et al., 1996), dielectrics (Lan et al., 2017), quantum cascade laser(Szymański et al., 2015) and semiconductors(Hamster et al., 1993) are not efficient enough as emitted radiations are of narrow bandwidth and low efficiency. Laser-plasma interaction can produce highly energetic and ultra-short $\mathrm{THz}$ fields. Laser-plasma based methods (Malik and Malik, 2011; Singh and Malik, 2014; Varshney et al., 2015; Varshney et al., 2018; Cho et al., 2015; Gurjar et al., 2020) have been used for high power THz emission in past few years as plasmas have no damage threshold limit. As laser technology progresses for higher peak intensities (Strickland and Mourou, 1985), THz peak power can be increased by scaling up the laser intensity.

Laser induced wakefield comprises electrostatic and electromagnetic components in the presence of a transverse magnetic field. The magnetized wakefield has nonzero group velocity that allows wake to propagate in underdense plasmas and emits electromagnetic radiation at the a plasma-vacuum boundary. $\mathrm{THz}$ radiation emits through laser generated wakefield in the presence of a transverse magnetic field in Cherenkov wake radiation mechanism (Yoshii et al., 1997). Amplitude of $\mathrm{THz}$ radiation expressed as $\left(\omega_{c} / \omega_{p}\right)$ (ratio of electron cyclotron frequency and plasmonic frequency) times the wakefield amplitude. Linear-mode conversion(Sheng et al., 2005b) has been another way to produce $\mathrm{THz}$ radiation in non-uniform plasmas, where laser wakefield is converted into electromagnetic radiation with conversion efficiency scaling as $\sim\left(\frac{\omega_{T}}{\omega_{L}}\right)^{3}$, where $\omega_{T}$ and $\omega_{L}$ are the terahertz and laser wave frequency, 
respectively. Properties of $\mathrm{THz}$ radiation can be tuned using the laser intensity and the scalelength of the non-uniform plasmas(Sheng et al., 2005a). Nonlinear mixing of laser beat wave with non-uniform plasmas can generate $\mathrm{THz}$ fields under appropriate phase matching conditions (Singh and Sharma, 2013; Varshney et al., 2018). THz radiation has also been reported in air and noble gas by using two color lasers(Saxena et al., 2018).

Nanoparticles (NP's) show high-order nonlinearity relative to the collective oscillations of relatively free-electrons at plasmon frequency, which makes NPs a potential candidate for nano-electronic, nano-optic, nano-photonics, and biomedical applications. Heating of the electrons in the NPs array allows coherent $\mathrm{THz}$ pulse generation that can be tuned under the variation of the geometries of the metallic NPs(Fadeev et al., 2018). Electron photoemission and ponderomotive acceleration through surface enhanced optical fields can produce $\mathrm{THz}$ radiation from gold nano-rods when illuminated by intense laser pulses with different central wavelength (Takano et al., 2019). THz pulses are generated through the acceleration of the ejected electrons under the influence of ponderomotive force that arises from the inhomogeneous plasmon field when femtosecond laser pulses interact with the array of silver nanoparticles. $\mathrm{THz}$ emission can be controlled on the basis of metal nanoparticle morphology(Polyushkin et al., 2011). Semiconducting and graphite NPs exhibit collective oscillations at low plasmon frequency in THz range while other metallic NP's have frequency out of the THz range. Therefore, graphite NPs can be explored for the THz radiation generation and related application(Varshney et al., 2020). In the present study, we propose to investigate the induced $\mathrm{THz}$ field from the shaped laser beam interaction with a mixture of NPs in Argon gas [see schematics in Fig. 1]. Shape of the NPs can be tailored to tune the plasmon frequency and therefore, the properties of the $\mathrm{THz}$ field. Different types of laser intensity profiles have been employed to enhance the $\mathrm{THz}$ field from nano-particle interaction. 
This work is based on the laser intensity profile effect on $\mathrm{THz}$ field generation via nano-particle dynamics. The laser intensity distribution is varied for different laser electric field profile (see in fig.2). We consider different plasmon resonance conditions for different shapes of the nanoparticles. Different restoring forces for both spherical and cylindrical NPs that lead to different plasmon resonance conditions have been considered for accurate $\mathrm{THz}$ field estimation. THz field attains peak value when laser beat frequency $\left(\omega=\omega_{1}-\omega_{2}\right)$ approaches the resonance. Energy loss mechanism has also been considered in this formulation with a damping factor $(\Gamma)$. Contribution of the non-conducting and the bound electron of the background is being included in the calculations of the effective permittivity. THz field has been estimated and discussed for varying shape of laser field envelope, basal plane spacing and laser spot size. Finally, results are summarized in the last Sec.

\section{Physical Model and THz Field Calculation}

We consider the nonlinear mixing of lasers in plasma embedded by nanoparticles for emission of $\mathrm{THz}$ radiation. Schematic of the physical mechanism is shown in fig. 1 . We consider two linearly polarized cosh-Gaussian laser pulses co-propagating through a mixture of NPs in argon gas. Electric field profile corresponding to the laser pulses is defined as (Purohit and Gaur, 2019; Hricha et al., 2021)

$$
\vec{E}_{j}=\hat{y} E_{0} \cosh \left(\frac{y b}{w_{0}}\right) e^{-y^{2} / w_{0}^{2}} e^{-\mathrm{i}\left(\omega_{j} t-k_{j} z\right)},
$$

where $j=1,2$ represents two different lasers, $w_{0}$ is the laser beam-width, $E_{0}$ is the laser electric field amplitude. Variation in the parameter $(b)$ changes the field envelope of the laser e.g. Gaussian beam $(b=0)$, Cosh Gaussian $(b=1)$, flat-top Gaussian $(b=1.45)$ and ring shape $(b=2)$. Intensity redistribution related to the above mentioned field profile of lasers is shown in Fig. 2. Here, we consider the geometries of spherical and cylindrical geometries of NPs with 
their basal plane (plane perpendicular to the principal axis in the crystal systems) oriented in two different directions, i.e., normal to the electric field $\vec{E}_{j} \| k_{i(=z)}$ and parallel to the electric field $\vec{E}_{j} \perp k_{i(=y)}$, where $k_{i}$ is the symmetry axis normal to the basal plane with different orientations within the nanoparticle.

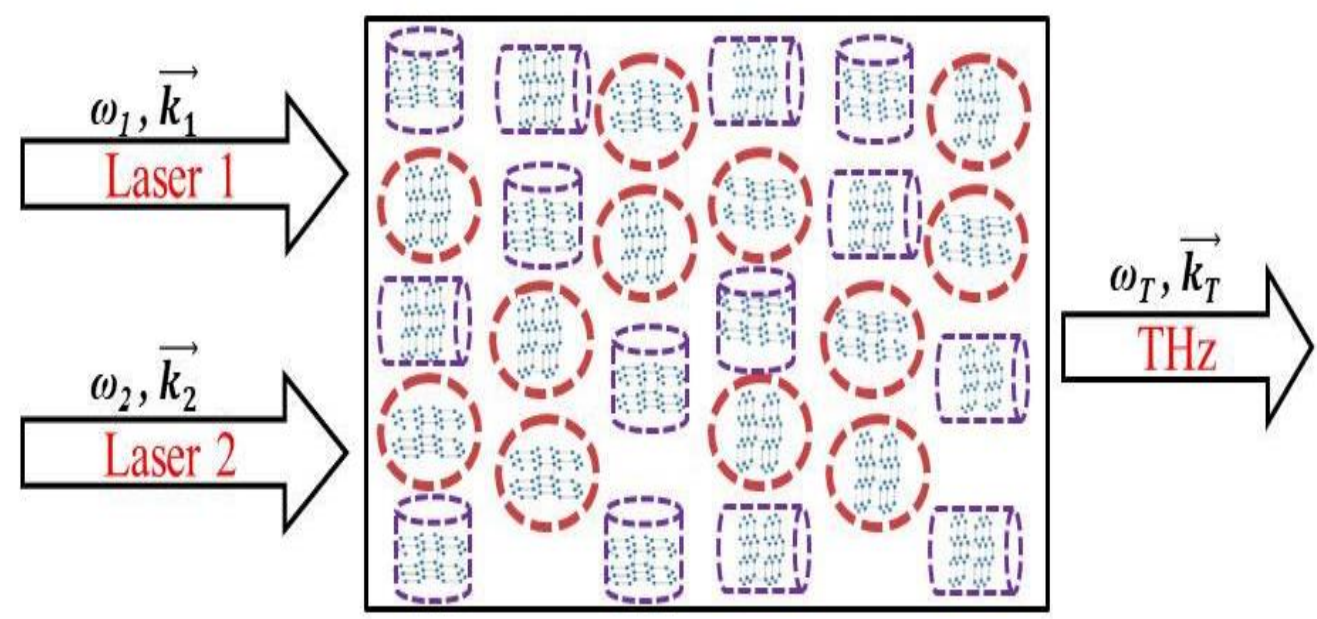

Fig. 1. Schematic representation of THz field generation from laser beat-wave interaction with nanoparticle embedded argon gas.

Since the mixture of SNPs and CNPs is considered in host Ar gas, the total macroscopic density of nanoparticles is expressed as

$$
n_{0}=\sum_{k_{i}}\left(n_{s}+n_{c}\right)=\sum_{k_{i}} n_{0, k_{i}}\left(g_{s k_{i}}+g_{c k_{i}}\right),
$$

Where $n_{s}$ and $n_{c}$ represents the densities of SNPs and CNPs, respectively. Particle anisotropy is an important factor that leads to self-assembly of NPs in a specific shape. Experimentally, wet chemical methods have been used widely for production of spherical and cylindrical metal nanoparticles (Sharma et al., 2020; Guerrero-Martínez, 2011). The factors $g_{s k_{i}}=4 \pi r_{s k_{i}}^{3} / 3 d_{s k_{i}}^{3}$ and $g_{c k_{i}}=\pi r_{c k_{i}}^{2} h_{c k_{i}} / d_{c k_{i}}^{3}$ represent the volume fraction, i.e. the volume occupied by SNPs and CNPs divided by volume of the unit cell for each of the directions (perpendicular and parallel), 
where $d_{s k_{i}}, d_{c k_{i}}$ and $r_{s k_{i}}, r_{c k_{i}}$ are the average distance and radii between two consecutive cylindrical nanoparticles and spherical nanoparticles in a given direction, respectively.

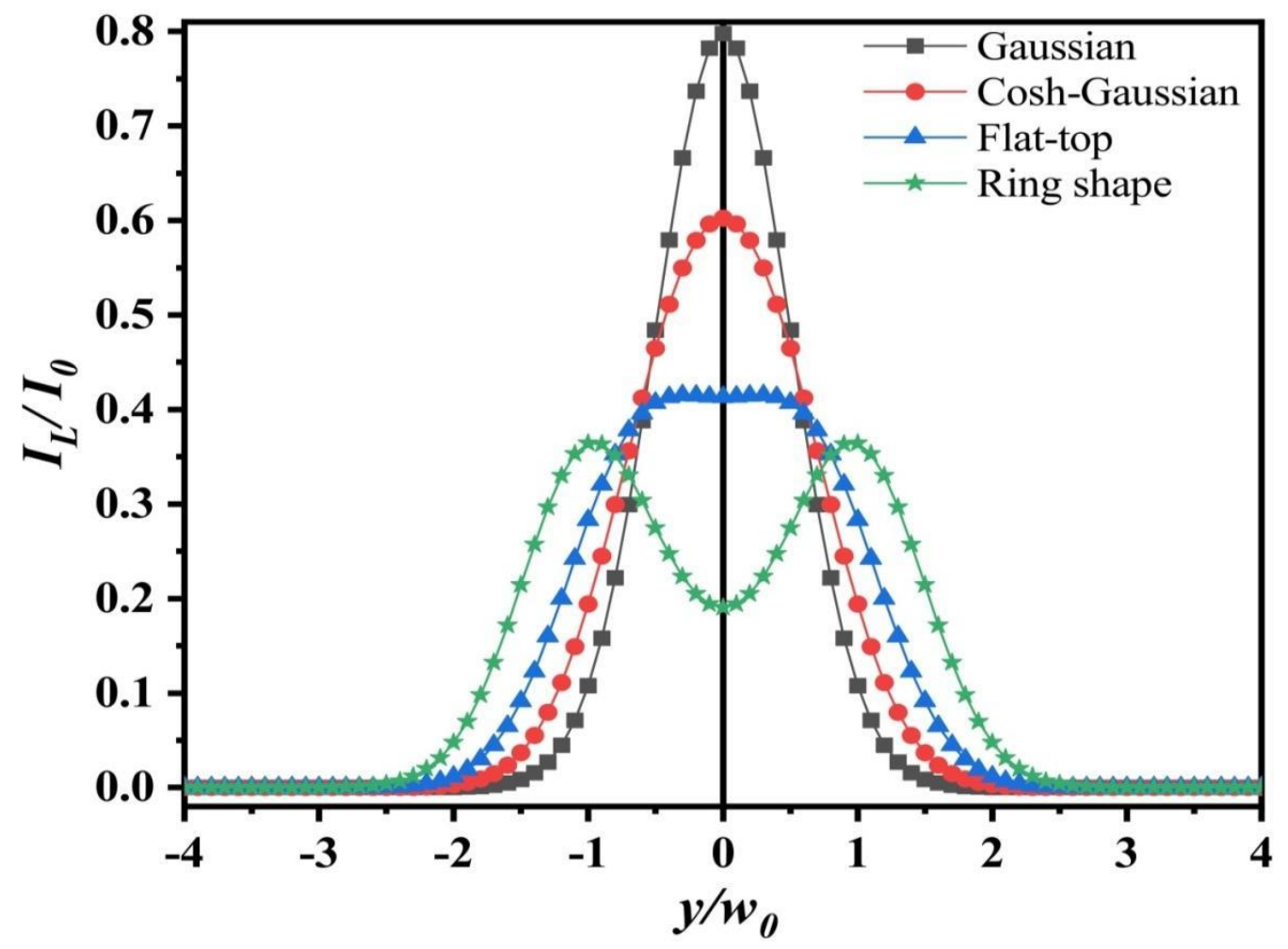

Fig. 2. Intensity distribution corresponding to laser field profile for Gaussian (black), Cosh Gaussian (red), Flat-top Gaussian (blue) and Ring shape (green) pulses.

Here, we are assuming that charges are distributed evenly around the nanoparticles and the modulated density of CNPs and SNPs can be written as

$n^{\prime}=n_{\alpha} e^{i \alpha z}$,

where $n_{\alpha}$ and $\alpha$ are the amplitude and the wave number of the density ripples.

Density modulation of nanoparticles in the above mentioned pattern can be produced with the stream of nanoparticles through nozzle in the background host Ar gas. The motion of spherical and cylindrical electron clouds corresponding to NPs can be compared with the 
motion of a single electron in a laser electric field. Therefore, nonrelativistic electron cloud dynamics can be expressed in the form of an electron's equation of motion as

$$
\frac{d \overrightarrow{v_{j}}}{d t}+\delta_{G e n} \overrightarrow{x_{j}}+\Gamma \overrightarrow{v_{j}}=-\frac{e}{m} \overrightarrow{E_{j}}
$$

where $m$ is the mass of electron, $e$ is the electron charge, $\Gamma$ is the damping factor and $\omega_{p}=\left(4 \pi n_{0} e^{2} / m\right)^{1 / 2}$ is electron plasma frequency. Damping mechanisms during laser beat wave interaction with clustered plasma include electron-electron, electron-phonon and electron-surface scattering. The term $\delta_{G e n} x_{j}$ represents the generalised restoration force exerted by immobile ions, which helps to return the electronic cloud to its equilibrium state in the presence of a laser electric field. For the spherical nanoparticles $\delta_{G e n}=\delta_{s p}=\omega_{p}^{2} / 3$, the cylindrical nanoparticles oriented with perpendicular direction of wave propagation $\delta_{G e n}=\delta_{c y \perp}=\omega_{p}^{2} / 2$ and cylindrical nanoparticles oriented with parallel direction of wave propagation $\delta_{G e n}=\delta_{c y \|}=\omega_{p}^{2}$. Oscillatory velocity for the electrons can obtained from Eq. (4) as

$$
\overrightarrow{v_{j}}=\frac{-i e \omega_{j} \overrightarrow{E_{j}}}{m\left(\omega_{j}^{2}+i \Gamma \omega_{j}-\delta_{G e n}\right)},
$$

The ponderomotive force $\left(\vec{F}_{N L}\right)$ is a non-linear force that imparts on conduction electrons at the beat frequency $\omega=\omega_{1}-\omega_{2}$ by the inhomogeneous electric field of laser beat wave. Ponderomotive force can be estimated using the standard formulation of ponderomotive potential $\phi_{p}=-m \vec{v}_{1} * \vec{v}_{2}^{*} / 2 e$ as 


$$
\begin{aligned}
\overrightarrow{F_{p}^{N L}}= & \frac{-e^{2} \omega_{1} \omega_{2} E_{0}^{2}}{2 m} * \\
& \frac{\left\{-\frac{2 y}{w_{0}^{2}}+\frac{b}{w_{0}} \sinh \left(\frac{2 y b}{w_{0}}\right)-\frac{2 y}{w_{0}^{2}} \cosh \left(\frac{2 y b}{w_{0}}\right) \hat{y}-i k^{\prime} \hat{z}\right\} \exp \left[-2 y^{2} / w_{0}^{2}\right]^{*} \exp \left[i\left(k^{\prime} z-\omega t\right)\right]}{\left(\omega_{1}^{2}+i \Gamma \omega_{1}-\delta_{\text {Gen }}\right)\left(\omega_{2}^{2}+i \Gamma \omega_{2}-\delta_{\text {Gen }}\right)}
\end{aligned}
$$

Further, one can write equation of motion under the effect of ponderomotive force as

$$
\frac{\partial^{2} \overrightarrow{x^{N L}}}{\partial t^{2}}+\delta_{G e n} \overrightarrow{x^{N L}}+\Gamma \frac{\partial \overrightarrow{x^{N L}}}{\partial t}=\frac{\overrightarrow{F_{p}^{N L}}}{m}
$$

where, $\vec{x}^{N L}$ is the displacement of the electrons. Y-component of the nonlinear velocity $\vec{v}^{N L}$ of the electrons can be obtained by solving Eq. (7) as

$$
\begin{aligned}
v_{y}^{N L}= & \frac{i e^{2} \omega_{1} \omega_{2} \omega E_{0}^{2}}{2 m^{2}} * \\
& \frac{\left\{-\frac{2 y}{w_{0}^{2}}+\frac{b}{w_{0}} \sinh \left(\frac{2 y b}{w_{0}}\right)-\frac{2 y}{w_{0}^{2}} \cosh \left(\frac{2 y b}{w_{0}}\right)\right\} \exp \left[-2 y^{2} / w_{0}^{2}\right]^{*} \exp \left[i\left(k^{\prime} z-\omega t\right)\right]}{\left(\omega^{2}+i \Gamma \omega-\delta_{G e n}\right)\left(\omega_{1}^{2}+i \Gamma \omega_{1}-\delta_{G e n}\right)\left(\omega_{2}^{2}+i \Gamma \omega_{2}-\delta_{\text {Gen }}\right)}
\end{aligned}
$$

Hence, the nonlinear macroscopic current density is evaluated by taking the contribution of both types of oriented NPs as

$$
J_{y}^{N L}=\sum_{k_{i}}\left(\delta_{1} * g_{s k_{i}}+\delta_{2} * g_{c k_{i \perp}}+\delta_{3} * g_{c k_{i \|}}\right) n^{\prime} e v_{y}^{N L},
$$

where, $\delta_{1}, \delta_{2} \& \delta_{3}$ are the arbitrary constants considered to include the individual contribution of spherical, cylindrical and mixture of spherical \& cylindrical nanoparticles. 


$$
\begin{aligned}
& \left(J_{y}^{N L}\right)=\frac{i e^{3} \omega_{1} \omega_{2} \omega n_{\alpha} E_{0}^{2}}{2 m^{2}} * \exp \left[i\left\{\left(k^{\prime}+\alpha\right) z-\omega t\right)\right]^{*} \\
& \left\{-\frac{2 y}{w_{0}^{2}}+\frac{b}{w_{0}} \sinh \left(\frac{2 y b}{w_{0}}\right)-\frac{2 y}{w_{0}^{2}} \cosh \left(\frac{2 y b}{w_{0}}\right)\right\} \exp \left[\frac{-2 y^{2}}{w_{0}^{2}}\right] * \\
& \left\{\begin{array}{c}
\frac{\delta_{1}\left(\frac{-4 \pi r_{s k_{i}}^{3}}{3 d_{s k_{i}}^{3}}\right)}{\left(\omega^{2}+i \Gamma \omega-\omega_{P}^{2} / 3\right)\left(\omega_{1}^{2}+i \Gamma \omega_{1}-\omega_{P}^{2} / 3\right)\left(\omega_{2}^{2}+i \Gamma \omega_{2}-\omega_{P}^{2} / 3\right)}+ \\
\frac{-\pi r_{c k_{i}}^{2} h_{c k_{i}} *}{d_{c k_{i}}^{3}}\left\{\begin{array}{l}
\frac{\delta_{2}}{\left(\omega^{2}+i \Gamma \omega-\omega_{P}^{2} / 2\right)\left(\omega_{1}^{2}+i \Gamma \omega_{1}-\omega_{P}^{2} / 2\right)\left(\omega_{2}^{2}+i \Gamma \omega_{2}-\omega_{P}^{2} / 2\right)} \\
+\frac{\delta_{3}}{\left(\omega^{2}+i \Gamma \omega-\omega_{P}^{2}\right)\left(\omega_{1}^{2}+i \Gamma \omega_{1}-\omega_{P}^{2}\right)\left(\omega_{2}^{2}+i \Gamma \omega_{2}-\omega_{P}^{2}\right)}
\end{array}\right\}
\end{array}\right\}
\end{aligned}
$$

SNPs follow radial symmetry, while CNPs have cylindrical symmetry. Therefore, CNPs are considered to be oriented in parallel and perpendicular to the laser polarization. Expression for nonlinear current density can be estimated using Eqs. (8) and (9) and described in Eq. (10).

The THz field wave equation is obtained from Maxwell's equations, as given below

$$
\nabla^{2} \vec{E}_{T H z}-\vec{\nabla} \cdot\left(\vec{\nabla} \cdot \vec{E}_{T H z}\right)+\frac{\omega^{2}}{c^{2}}\left(\varepsilon_{e f f} \cdot \vec{E}_{T H z}\right)=-\frac{4 \pi i \omega}{c^{2}} \vec{J}^{N L}
$$

where, $\varepsilon_{e f f}$ is the effective electric permittivity tensor of the medium of cylindrical and spherical graphite nanoparticles. Majorly, the conduction electrons of the graphite NPs give rise to nonlinearity. Effective permittivity of the medium needs to be calculated that consider both conducting and non-conducting bound electrons of the medium and, therefore, mathematical expression for the effective permittivity can be written as(Sau and Rogach, 2012)

$$
\left(\varepsilon_{\text {eff }}\right)_{G e n}=\varepsilon_{i}^{b}+\varepsilon^{f}=\varepsilon_{i}^{b}+1-\frac{\omega_{p}^{2}}{\left(\omega^{2}+i \Gamma \omega-\delta_{G e n}\right)} .
$$


Since it contains two separate shapes of graphite nanoparticles of different orientations, the efficient permittivity of the host medium is altered. Due to the introduction of SNPs and CNPs, the contribution of bound electrons to efficient permittivity can be rewritten as $\varepsilon_{i}^{b}=\varepsilon(s)+\varepsilon(c) / 2$, where

$$
\begin{gathered}
\varepsilon(s)=\varepsilon_{h}+3 \varepsilon_{h} \frac{\sum_{k_{i}} g_{s k_{i}} \frac{\varepsilon_{k_{i}}-\varepsilon_{h}}{\varepsilon_{k_{i}}+2 \varepsilon_{h}}}{1-\sum_{k_{i}} g_{s k_{i}} \frac{\varepsilon_{k_{i}}-\varepsilon_{h}}{\varepsilon_{k_{i}}+2 \varepsilon_{h}}} \\
\varepsilon(c)=\varepsilon_{h}+\sum_{k_{i}} g_{c k_{i}} \frac{\left(\varepsilon_{k_{i}}-\varepsilon_{h}\right)\left(\varepsilon_{k_{i}}+5 \varepsilon_{h}\right)}{\left(3-2 g_{c k_{i}}\right) \varepsilon_{k_{i}}+\left(3+2 g_{c k_{i}}\right) \varepsilon_{h}},
\end{gathered}
$$

where $\varepsilon_{h}$ is the permittivity of host medium and $\varepsilon_{k_{i}}$ is the permittivity of bound electrons (only) for different orientations of nanoparticles.

Expression for the $\mathrm{THz}$ field can be obtained taking the divergence of Eq. (11) and considering the assumption $v^{2}<\omega^{2}$

$$
\vec{E}_{T H z}=-\frac{4 \pi i}{\omega \varepsilon_{\text {eff }}} \vec{J}^{N L}
$$

Substituting Eq. (10) into Eq. (15), one can obtain the expression for the normalized $\mathrm{THz}$ radiation fields under consideration of only spherical nanoparticles $\left(\delta_{1}=1, \& \delta_{2}=\delta_{3}=0\right)$, only cylindrical nanoparticles $\left(\delta_{1}=0, \& \delta_{2}=\delta_{3}=1\right)$, and the mixture of spherical and cylindrical nanoparticles $\left(\delta_{1}=\delta_{2}=\delta_{3}=1\right)$ : 


$$
\begin{aligned}
& \left|\frac{E_{T H z}}{E_{0}}\right|=\frac{e \omega_{1} \omega_{2} n_{\alpha} E_{0} \omega_{p}^{2}}{2 m n_{0}} *\left\{-\frac{2 y}{w_{0}^{2}}+\frac{b}{w_{0}} \sinh \left(\frac{2 y b}{w_{0}}\right)-\frac{2 y}{w_{0}^{2}} \cosh \left(\frac{2 y b}{w_{0}}\right)\right\} * \exp \left[\frac{-2 y^{2}}{w_{0}^{2}}\right] *
\end{aligned}
$$

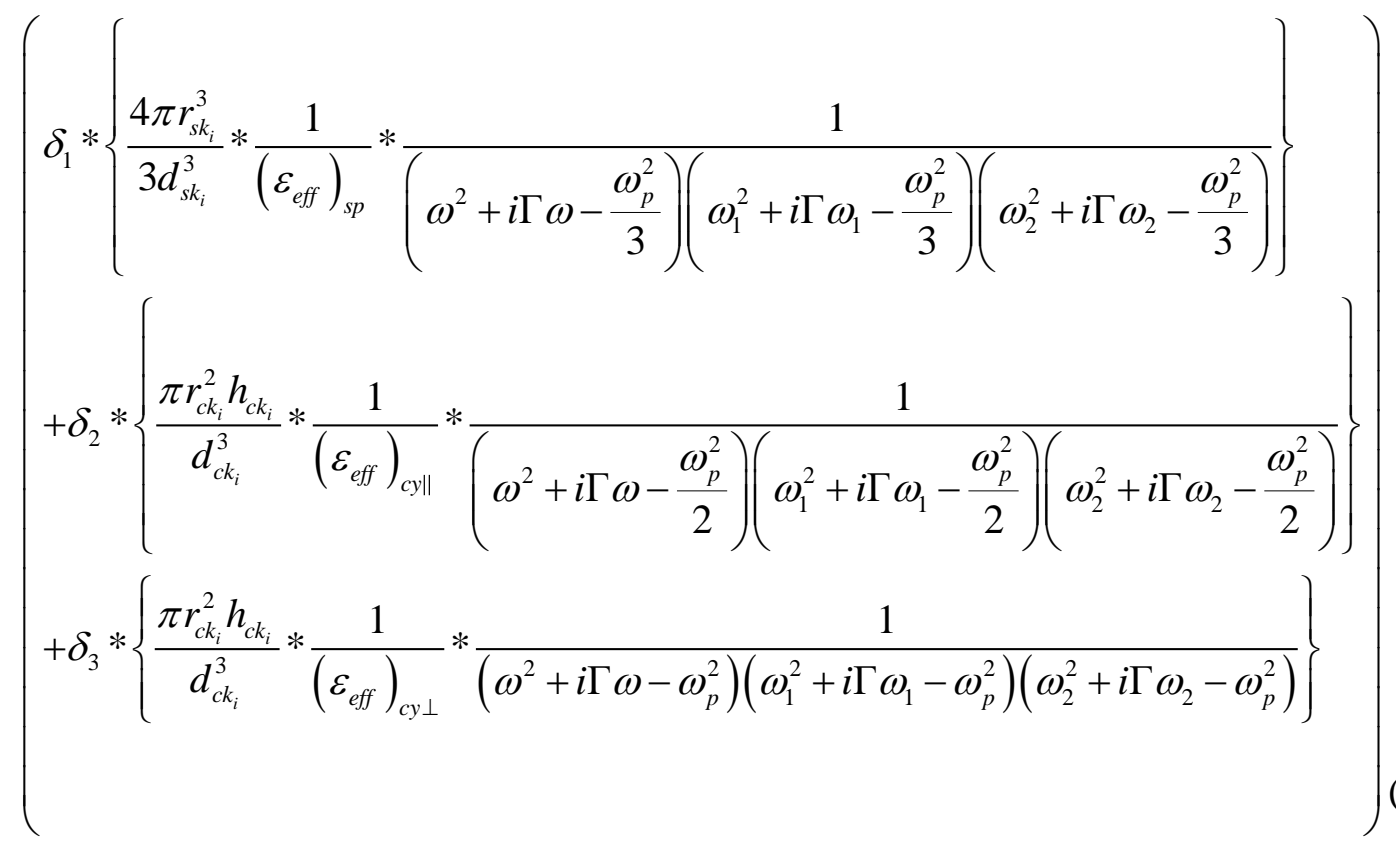

Number density of the embedded nanoparticles has been considered same while estimating the normalised THz field amplitude in Eq. (16). The efficiency of the THz radiation generation can be estimated by calculating the ratio of the energies of $\mathrm{THz}$ radiation to the incident laser (pump). Conversion efficiency of the $\mathrm{THz}$ emission in present physical mechanism can be expressed as $\eta=W_{T H z} / W_{\text {pump }}$, where the average energy densities of the emitted radiation $W_{T H z}$ and the incident pump lasers $W_{\text {pump }}$ are,

$$
W_{\text {pump }}=\sqrt{\frac{\pi}{2}} \frac{\varepsilon_{0} E_{0}^{2}}{2} \frac{1}{\sqrt{\frac{1}{w_{0}^{2}}}}\left(1+e^{b^{2} / 2}\right)
$$




$$
\begin{aligned}
& W_{T H z}=\frac{\varepsilon_{0} E_{0}^{2}}{2} \sqrt{\frac{\pi}{2}} *\left(\frac{n_{\alpha}}{n_{0}}\right)^{2} \frac{e^{2} \omega_{1}^{2} \omega_{2}^{2} \omega_{p}^{4}}{4 m^{2}}\left[\frac{3-b^{2}+4 e^{b^{2} / 2}+\left(1+b^{2}\right) e^{2 b^{2}}}{2 *\left(1 / w_{0}^{2}\right)^{3 / 2} w_{0}^{4}}\right] *
\end{aligned}
$$

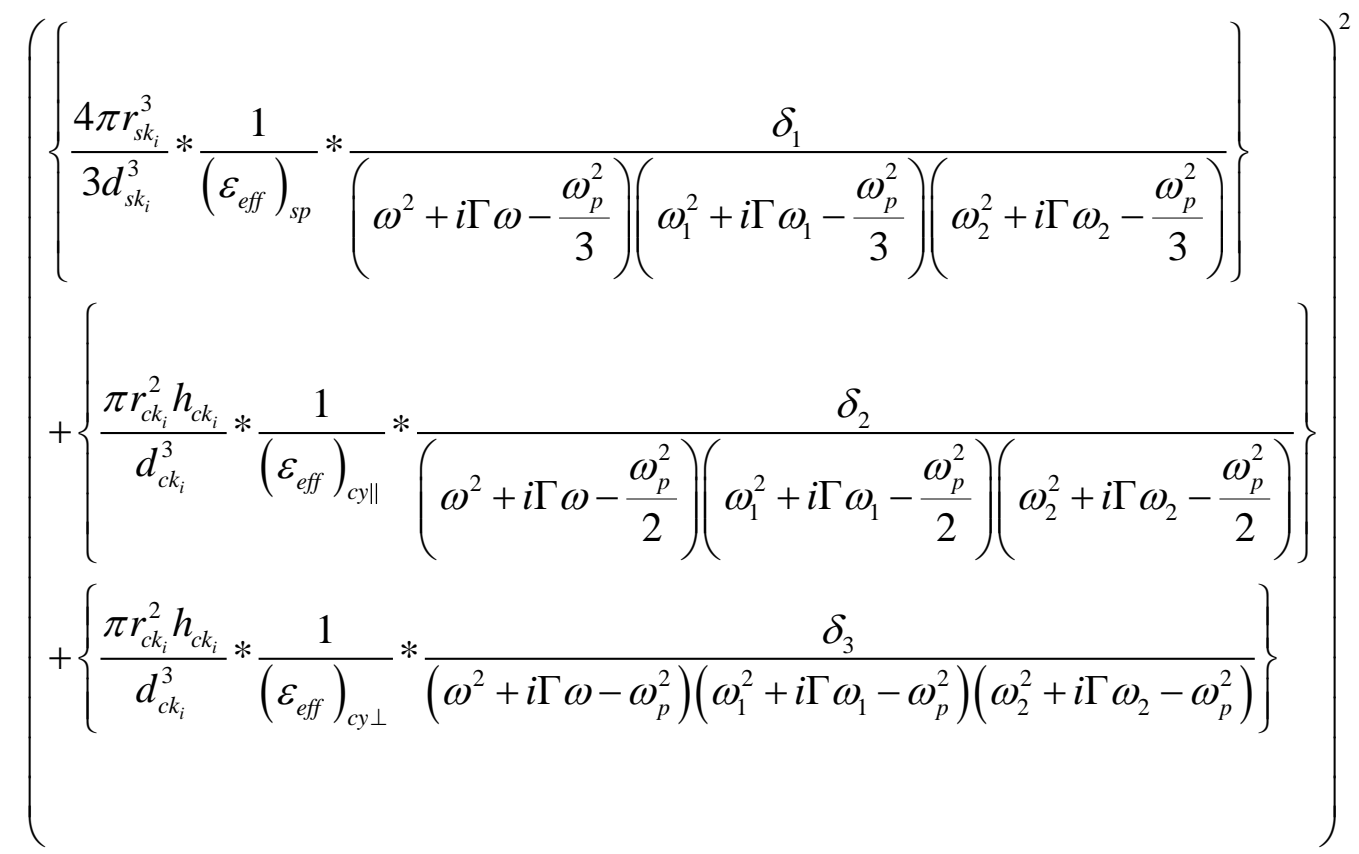

Eq. (19) describes the efficiency of $\mathrm{THz}$ radiation fields under consideration for three different cases (i) only spherical nanoparticles $\left(\delta_{1}=1, \& \delta_{2}=\delta_{3}=0\right)$ (ii) only cylindrical nanoparticles $\left(\delta_{1}=0, \& \delta_{2}=\delta_{3}=1\right)$, and (iii) the mixture of SNPs and CNPs $\left(\delta_{1}=\delta_{2}=\delta_{3}=1\right)$ 


$$
\begin{aligned}
& \eta=\left(\frac{n_{\alpha}}{n_{0}}\right)^{2} \frac{e^{2} \omega_{1}^{2} \omega_{2}^{2} \omega_{p}^{4}}{8 m^{2}}\left[\frac{3-b^{2}+4 e^{b^{2} / 2}+\left(1+b^{2}\right) e^{2 b^{2}}}{\left(1+e^{b^{2} / 2}\right) w_{0}^{2}}\right] *
\end{aligned}
$$

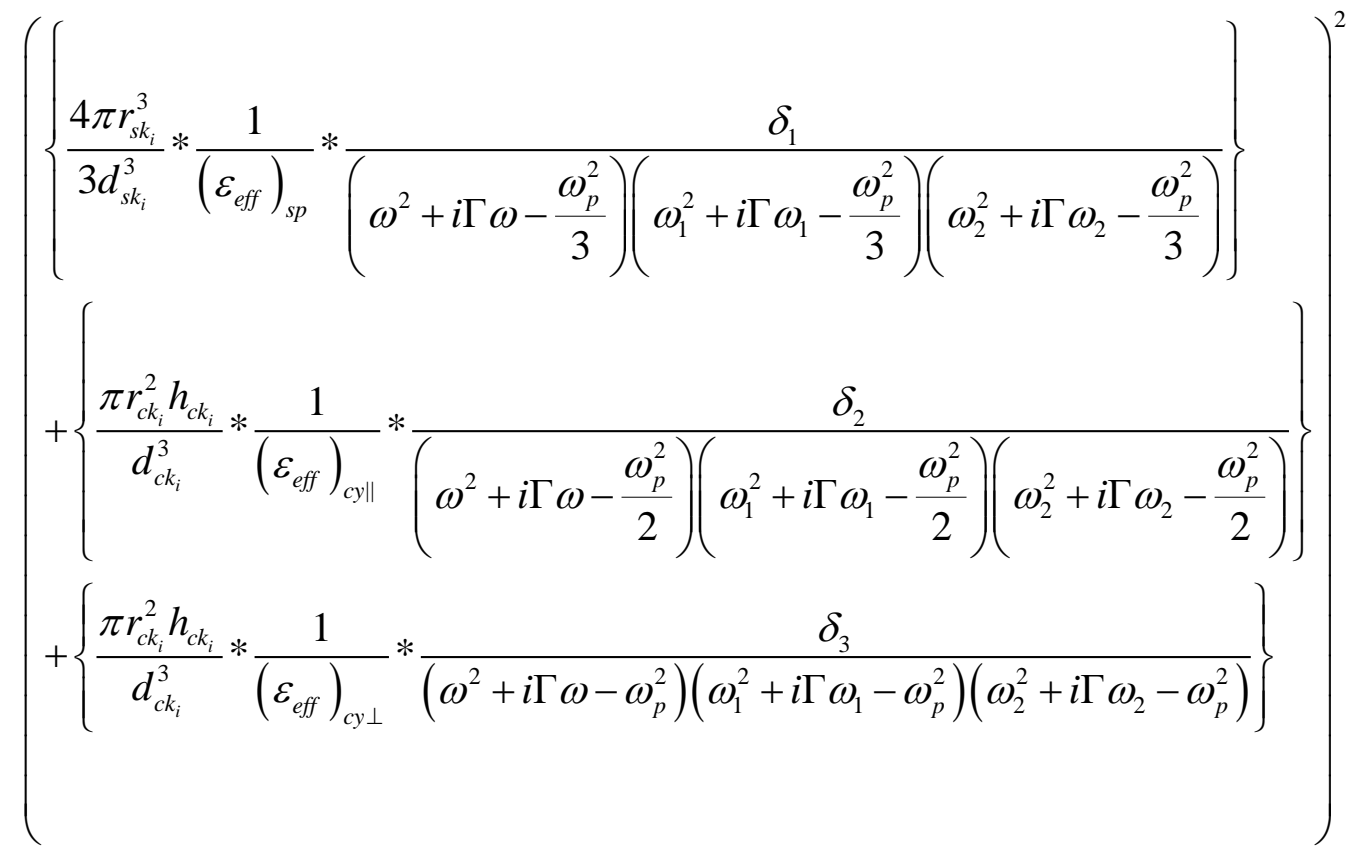

\section{Result and Discussion}

This section describes the numerical results obtained from the physical model for $\mathrm{THz}$ emission using nonlinear mixing phenomena, where laser beat wave envelope interacts with the density modulated mixture of SNPs and CNPs. Laser intensity distribution along the polarization direction affects the ponderomotive force strongly that influences the nonlinear transverse current. Since transverse current is a key source for radiation generation, laser intensity distribution is treated as a significant parameter in present numerical calculations to estimate the properties of $\mathrm{THz}$ radiations. $\mathrm{THz}$ field amplitude has been estimated as a function of $\mathrm{THz}$ frequency for variation in laser intensity distribution under different spatial field envelopes e.g. Gaussian, cosh-Gaussian, flat-Gaussian and ring shape. In present numerical calculations, we have considered $\mathrm{CO}_{2}$ laser with frequency $\omega_{1}=2 \times 10^{14} \mathrm{rad} / \mathrm{sec}\left(\lambda_{l}=\right.$ $10.1 \mu \mathrm{m})$, intensity $I=2 \times 10^{15} \mathrm{w} / \mathrm{cm}^{2}$ and spot size e.g. $10 \mu \mathrm{m}, 20 \mu \mathrm{m}, 30 \mu \mathrm{m}, 40 \mu \mathrm{m}$ respectively. Plasma frequency has been chosen as $\omega_{p}=2 \pi \times 10^{12} \mathrm{rad} / \mathrm{sec}$ corresponding 
to the plasma density $n_{0}=1.24 \times 10^{16} \mathrm{~cm}^{-3}$. Density modulation is considered as $30 \%$ of the background plasma density for individual NPs and mixture of NPs. Oscillatory velocity of electrons corresponding to above mentioned laser intensity is $v_{1}=0.3 c$. Number density of embedded NPs is the same while considering individual SNPs, CNPs or the mixture of NPs. Aspect ratio (height to width) is considered unity in present set of numerical calculations for CNPs. Transverse nonlinear current is the key source for the generation of THz field in laserplasma based methods. Fig. 3 shows the normalised THz amplitude as function of normalised frequency for spatially varying laser field envelope in case of SNPs, CNPs, and the mixture of SNPs and CNPs respectively. THz field attains peak value when laser beat frequency $(\omega=$ $\omega_{1}-\omega_{2}$ ) approaches the resonance. Plasmon oscillations resonates at the surface of SNPs when $\omega_{1}-\omega_{2}=\frac{\omega_{p}}{\sqrt{3}}$, while resonance arises at $\omega_{1}-\omega_{2}=\frac{\omega_{p}}{\sqrt{2}}$ and $\omega_{1}-\omega_{2}=\omega_{p}$ for CNPs in parallel and perpendicular orientation with laser propagation direction. The $\mathrm{THz}$ field decreases as one move away from the resonance.

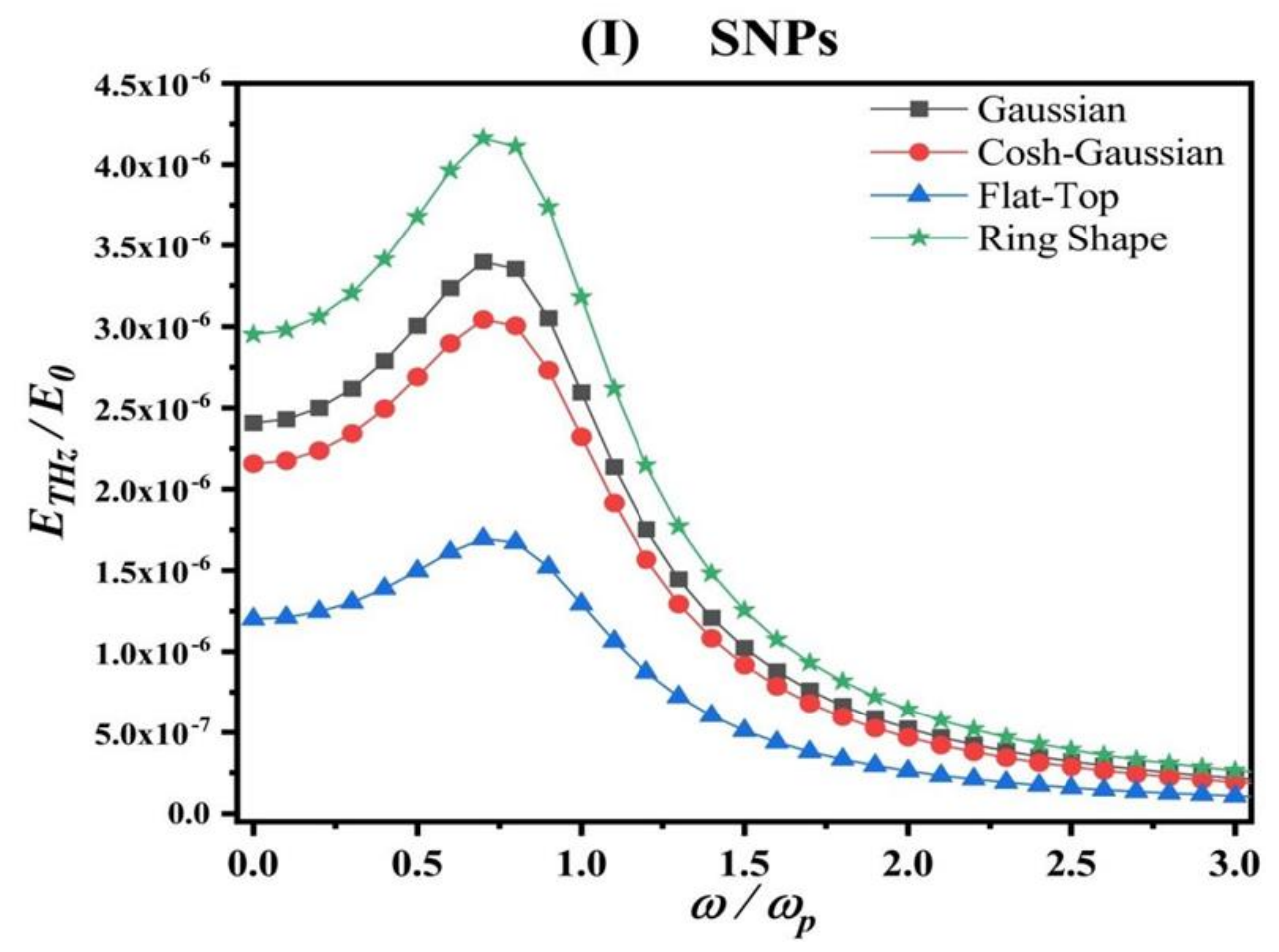




\section{(II) CNPs}

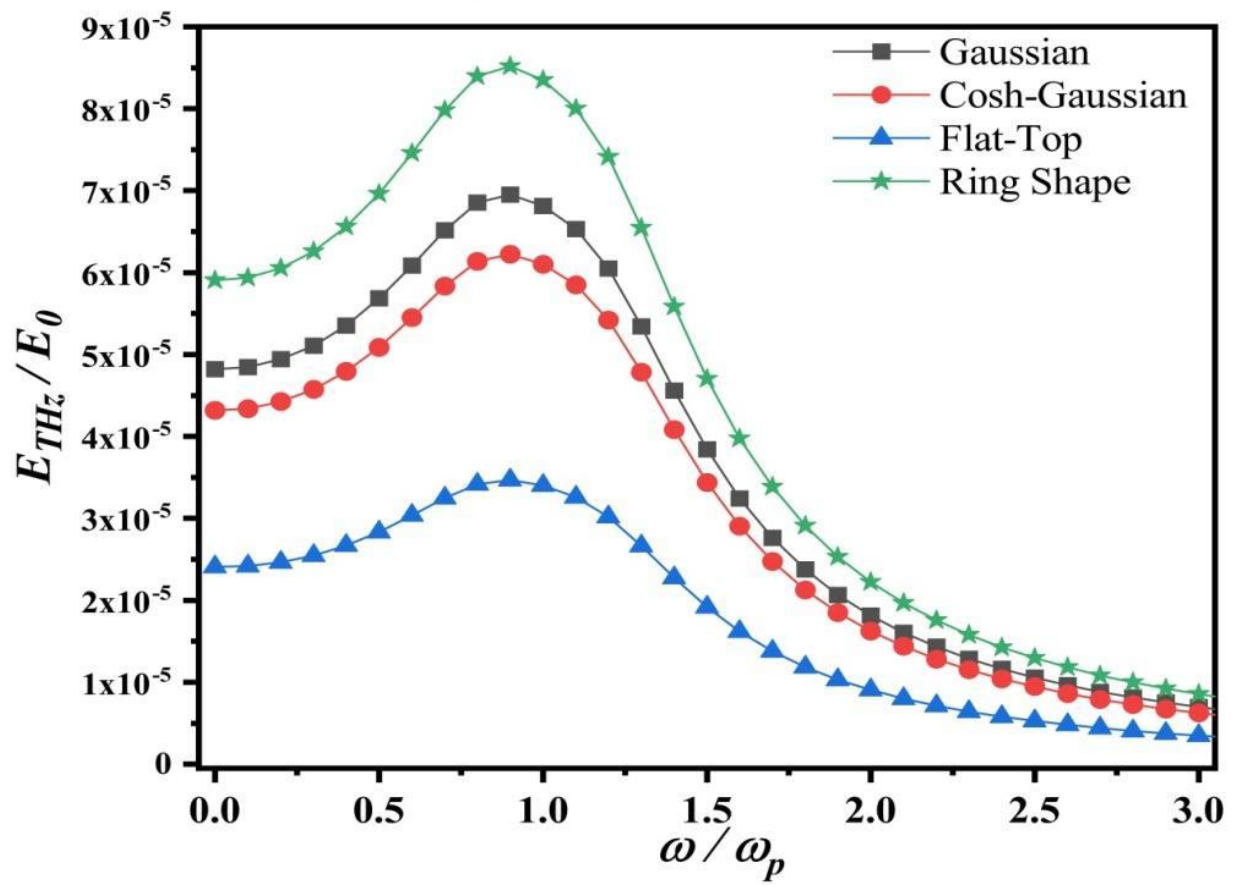

\section{(III) $\mathrm{SNPs}+\mathrm{CNPs}$}

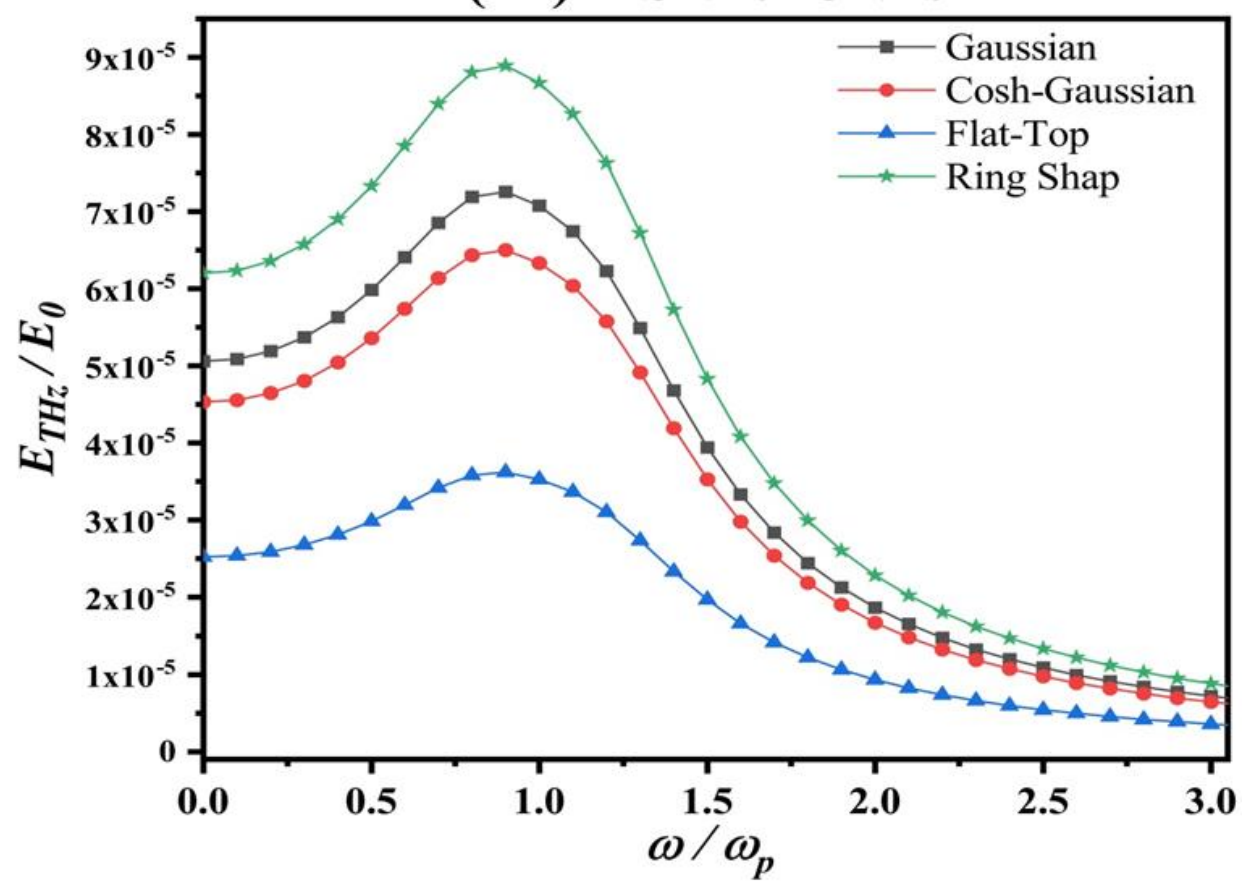

Fig. 3 Normalised THz amplitude $\left(E_{T H z} / E_{0}\right)$ with normalised terahertz frequency $\left(\omega / \omega_{p}\right)$ for various shaped laser pulse (I) SNPs (II) CNPs (III) Mixture of SNPs and CNPs. Laser-plasma parameter considered with frequency $\omega_{1}=2 \times 10^{14} \mathrm{rad} / \mathrm{sec}\left(\lambda_{l}=10.1 \mu \mathrm{m}\right)$, intensity $I=$ 
$2 \times 10^{15} \mathrm{w} / \mathrm{cm}^{2}$, plasma frequency $\omega_{p}=2 \pi \times 10^{12} \mathrm{rad} / \mathrm{sec}$ corresponding to the plasma density $n_{0}=1.24 \times 10^{16} \mathrm{~cm}^{-3}$.

Exact phase matching and resonant excitation of $\mathrm{THz}$ radiation is achieved by introducing density modulation with suitable ripple wave number. Exact matching of the ripple wave vector with the laser beat wave number provides the maximum transfer of energy and momentum for resonance conditions. Previously, Sharma et al. [20] estimated the THz field considering the same plasmon resonance condition for both SNPs and CNPs that is inappropriate to consider as the plasmon resonance condition depends on the symmetry of NPs. From Fig. 3(b), it is observed that CNPs contribute more towards the THz field in comparison to the SNPs, where the radius of both the NPs and the basal plane spacing is considered the same. Also, the resonance appears to shift [in Figs. 3(a) and 3(b)] towards the higher value of frequency in case of CNPs that indicate the variation in timescale of effective energy transfer from laser beat wave to CNPs in comparison to SNPs. Nonlinear current density enhances as the number of conduction electrons per unit volume increases for mixture of SPNs and CNPs. Therefore, the mixture of the SNPs and CNPs contributes more toward the THz field as shown in Fig. 3 (c). Here, variation in laser intensity distribution greatly affects the nonlinear current density as ponderomotive force enhances when laser intensity re-distributed along the polarization direction and therefore, $\mathrm{THz}$ field increases for ring shape field envelope. Significant enhancement observed in $\mathrm{THz}$ field amplitude for ring shape laser field envelope for mixture of SNPs and CNPs. Present numerical results are found to be consistent with the previously reported results by Javan and Erdi (Javan and Erdi, 2017), where laser beat wave interacts with density modulated SNPs under phase matching condition.

Since the graphite NPs are anisotropic in nature, orientation of the basal plane and related spacing is the key factor to consider in present numerical calculations. Basal plane 
spacing can also affect the THz field amplitude as number density of NPs varies with basal plane spacing $\left(d_{s}\right.$ or $\left.d_{c}\right)$. Therefore, the number of conduction electrons of the spherical and cylindrical cloud of electrons involved in plasmon excitation vary that would change the transverse nonlinear current. Fig. 4 shows the variation of $\mathrm{THz}$ field with basal plane spacing for ring shape laser field envelope in case of only SNPs and mixture of NPs (SNPs \& CNPs). $\mathrm{THz}$ field decreases for increasing value of basal plane spacing.

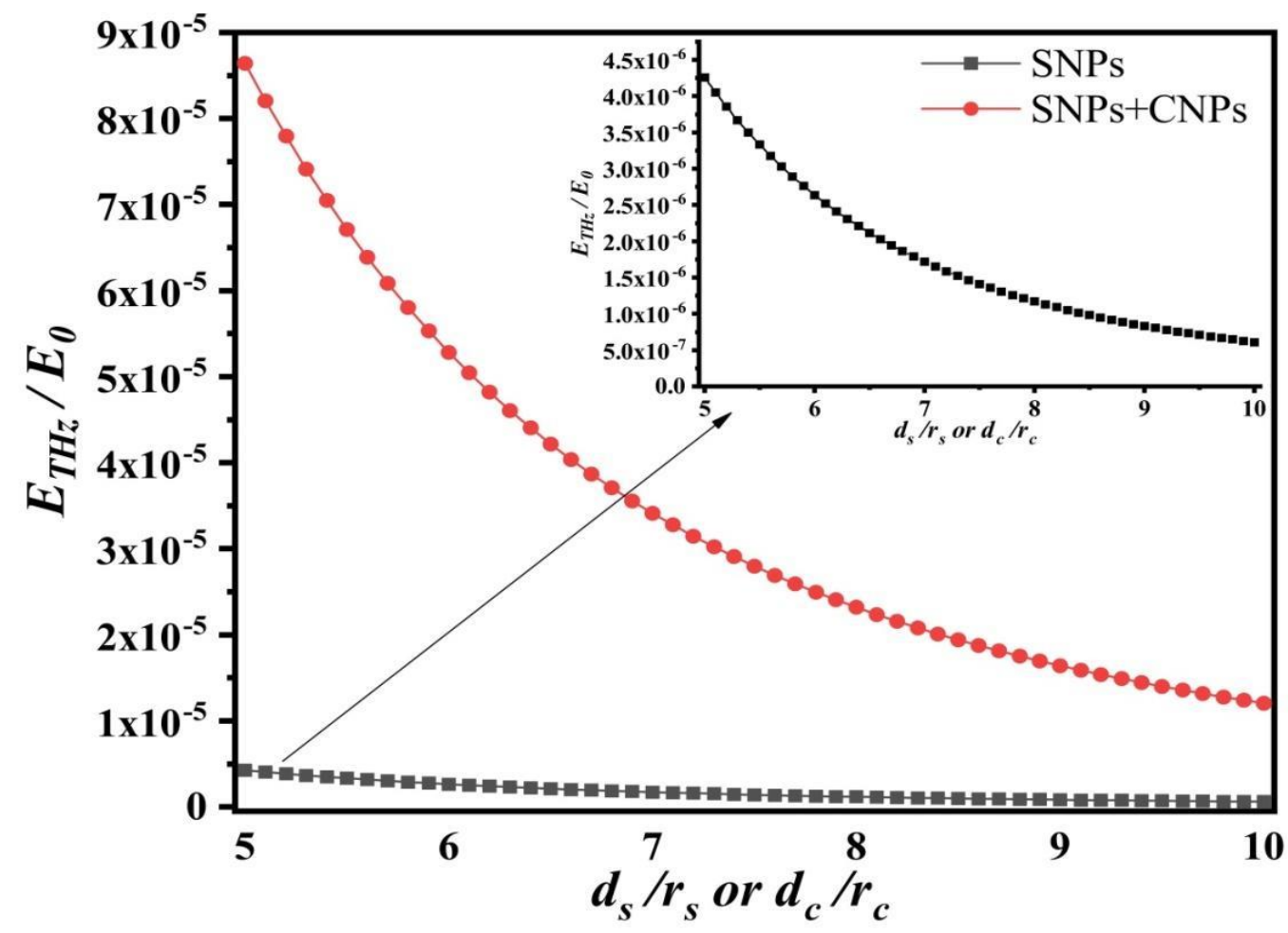

Fig. 4 Normalised THz amplitude $\left(E_{T H z} / E_{0}\right)$ as function of basal plane spacing for spherical, cylindrical and combination of spherical and cylindrical nanoparticles in case of ring shape field envelope. Laser-plasma parameter considered with frequency $\omega_{1}=2 \times 10^{14} \mathrm{rad} /$ $\sec \left(\lambda_{l}=10.1 \mu \mathrm{m}\right), \quad$ intensity $I=2 \times 10^{15} \mathrm{w} / \mathrm{cm}^{2}, \quad$ Plasma frequency $\omega_{p}=2 \pi \times$ $10^{12} \mathrm{rad} / \mathrm{sec} \mathrm{sec}$ corresponding to plasma density $n_{0}=1.24 \times 10^{16} \mathrm{~cm}^{-3}$.

Figure 5 show the THz field as a function of normalised frequency for varying laser beam width parameter. Ponderomotive force increases for smaller beam width as laser intensity is 
distributed in a small area that leads to maximum laser energy transfer to the NPs and hence there excite large amplitude surface plasmon. Therefore, nonlinear current density increases and hence THz amplitude enhances for decreasing value of the laser beam width.

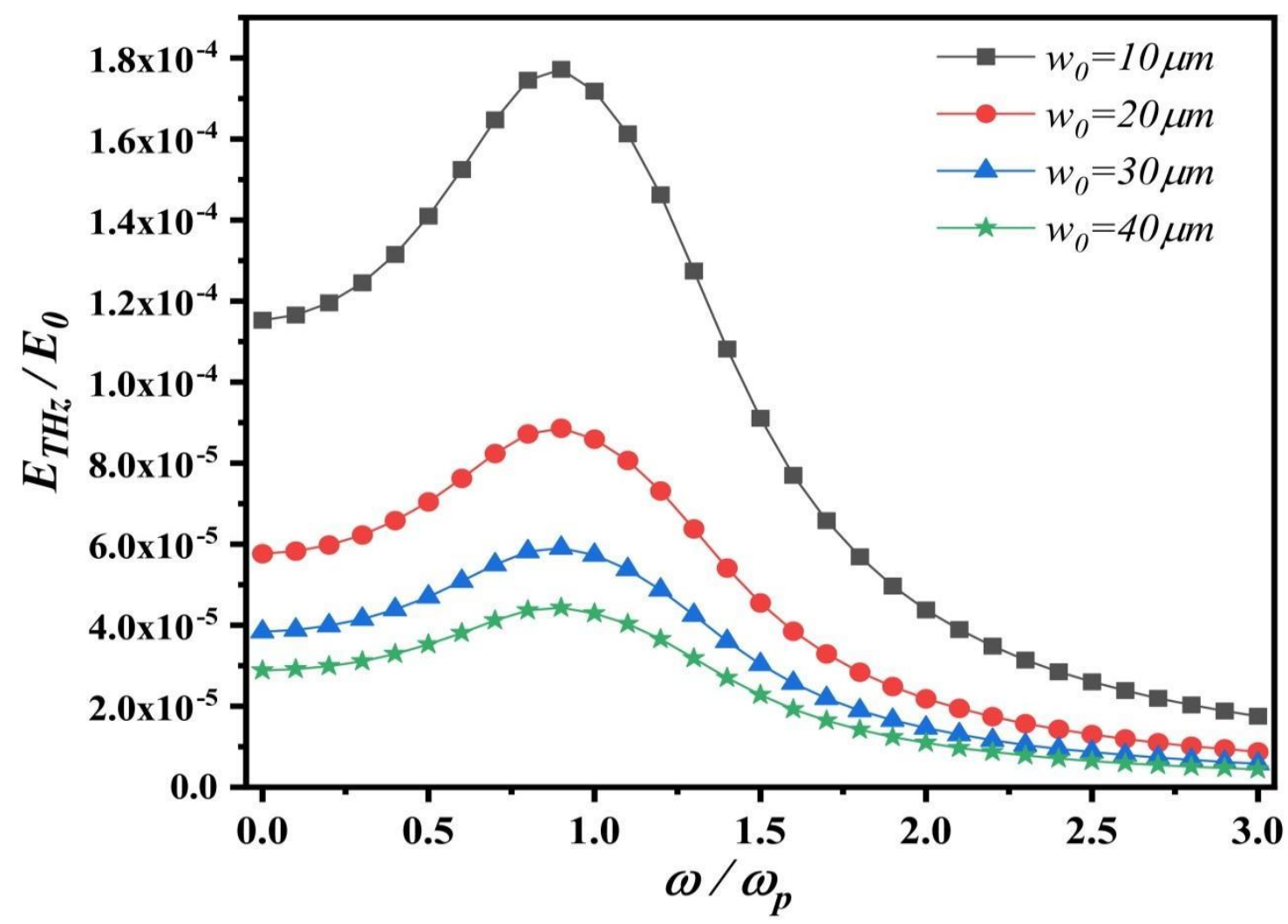

Fig. 5 Normalised THz amplitude $\left(E_{T H z} / E_{0}\right)$ as function of normalized terahertz frequency ( $\left.\omega / \omega_{p}\right)$ and laser beam width corresponding to ring shape field envelope. Laser-plasma parameter considered with frequency $\omega_{1}=2 \times 10^{14} \mathrm{rad} / \mathrm{sec}\left(\lambda_{l}=10.1 \mu \mathrm{m}\right)$, intensity $I=$ $2 \times 10^{15} \mathrm{w} / \mathrm{cm}^{2}$, Plasma frequency $\omega_{p}=2 \pi \times 10^{12} \mathrm{rad} / \mathrm{sec} \mathrm{sec}$ corresponding to plasma density $n_{0}=1.24 \times 10^{16} \mathrm{~cm}^{-3}$.

Efficiency $(\eta)$ for the present physical mechanism of $\mathrm{THz}$ emission has been derived in the physical model (see Eq. 19). Conversion efficiency of the order $10^{-12}$ estimated for spherical NPs in case of Gaussian pulses while it enhanced significantly and estimated as $10^{-6}$ for a mixture of spherical and cylindrical NPs using ring shape laser pulses. Laser energy transfers efficiently in $\mathrm{THz}$ field when intensity distribution is changing from Gaussian field 
envelope to ring shaped field envelope. Ponderomotive force changes significantly when laser intensity spread spatially. Here, we also estimated theoretically the power and energy of emitted THz radiation as $10^{3} \mathrm{Watt}$ and $5 \mathrm{n}$ Joule for incident laser pulse of $10^{8} \mathrm{Watt}$ and energy of $10 \mathrm{~mJ}$.

\section{Conclusion}

Laser intensity distribution plays a significant role in tuning the $\mathrm{THz}$ field generated from the laser beat wave interaction with a mixture of nanoparticles (Spherical and cylindrical) in argon gas. Plasmon resonance condition depends on the symmetry of the NPs. Therefore, surface plasmon resonates when $\omega_{1}-\omega_{2}=\frac{\omega_{p}}{\sqrt{3}}$ for SNPs, $\omega_{1}-\omega_{2}=\frac{\omega_{p}}{\sqrt{2}}$ and $\omega_{1}-\omega_{2}=\omega_{p}$ for CNPs in different orientations with the laser propagation direction. Non-linear transverse current increases as laser intensity redistributes spatially. $\mathrm{THz}$ field attains peak value when laser beat frequency $\left(\omega_{1}-\omega_{2}\right)$ approaches the resonance. Basal plane spacing and laser spot size also affects the THz field significantly. Present study concludes that a ring shape electric field envelope produces a higher THz field in comparison to the Gaussian, flat Gaussian and Cosh-Gaussian field envelope being used with the mixture of SNPs and CNPs in Ar gas.

\section{Acknowledgement}

The authors would like to thank Prof DN Gupta (Delhi University) for careful reading of the manuscript and providing the useful suggestions.

\section{Declaration of Competing Interest}

We declare that we do not have any commercial or associative interest that represents a conflict of interest in connection with the work submitted.

\section{Data availability statement}

The data that support the findings of this study are available from the corresponding author upon reasonable request. 


\section{References}

Cho, M.H., Kim, Y.K., Suk, H., Ersfeld, B., Jaroszynski, D.A. \& Hur, M.S.: Strong terahertz emission from electromagnetic diffusion near cutoff in plasma. New Journal of Physics 17, 043045 (2015). https://doi.org/10.1088/1367-2630/17/4/043045.

FADEeV, D.A., OladyshKin, I.V. \& Mironov, V.A.: Terahertz emission from metal nanoparticle array. Opt. Lett. 43, 1939-1942

(2018). https://doi.org/10.1364/ol.43.001939.

Faure, J., VAn Tilborg, J., Kaindl, R.A. \& LeEmans, W.P.: Modelling Laser-Based TableTop THz Sources: Optical Rectification, Propagation and Electro-Optic Sampling. Optical and Quantum Electronics 36, 681-697 (2004). https://doi.org/10.1023/B:OQEL.0000039617.85129.c2.

GUERRERO-MARTíNEZ, A.: Nanostars shine bright for you Colloidal synthesis, properties and applications of branched metallic nanoparticles. Current opinion in colloid \& interface

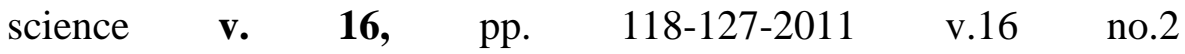
(2011). https://doi.org/10.1016/j.cocis.2010.12.007.

Gurjar, M.C., Gopal, K., Gupta, D.N., Kulagin, V.V. \& SuK, H.: High-Field Coherent Terahertz Radiation Generation From Chirped Laser Pulse Interaction With Plasmas. IEEE Transactions on Plasma Science 48, 3727-3734 (2020). https://doi.org/10.1109/tps.2020.3022903.

Hamster, H., Sullivan, A., Gordon, S., White, W. \& Falcone, R.W.: Subpicosecond, electromagnetic pulses from intense laser-plasma interaction. Physical Review Letters 71, 2725-2728 (1993). https://doi.org/10.1103/PhysRevLett.71.2725.

Hricha, Z., Halba, E.M.E., LAZREK, M. \& BelafHal, A.: Focusing properties and focal shift of a vortex cosine-hyperbolic Gaussian beam. Optical and Quantum Electronics 53, 449 (2021). https://doi.org/10.1007/s11082-021-03105-5.

JAVAN, N.S. \& ERDI, F.R.: Theoretical study of the generation of terahertz radiation by the interaction of two laser beams with graphite nanoparticles. Journal of Applied Physics 122, 223103 (2017). https://doi.org/10.1063/1.4995510.

Jepsen, P.U., Jacobsen, R.H. \& KeIDING, S.R.: Generation and detection of terahertz pulses from biased semiconductor antennas. J. Opt. Soc. Am. B 13, 2424-2436 (1996). https://doi.org/10.1364/josab.13.002424. 
LAN, C., BI, K., LI, B., ZHAO, Y. \& QU, Z.: Flexible all-dielectric metamaterials in terahertz range based on ceramic microsphere/ PDMS composite. Opt. Express 25, 29155-29160 (2017). https://doi.org/10.1364/oe.25.029155.

MaLiK, H.K. \& MALIK, A.K.: Tunable and collimated terahertz radiation generation by femtosecond laser pulses. Applied Physics Letters 99, 251101 (2011). https://doi.org/10.1063/1.3666855.

Polyushkin, D.K., Hendry, E., Stone, E.K. \& Barnes, W.L.: THz Generation from Plasmonic Nanoparticle Arrays. Nano Letters 11, 4718-4724 (2011). https://doi.org/10.1021/nl202428g.

Purohit, G. \& GAUR, B.: Self-focusing of cosh-Gaussian laser beam and its effect on the excitation of ion-acoustic wave and stimulated Brillouin backscattering in collisionless plasma. Optical and Quantum Electronics 51, 398 (2019). https://doi.org/10.1007/s11082-019-2119-y.

SaU, T.K. \& Rogach, A.L.: Complex-Shaped Metal Nanoparticles, John Wiley and Sons (2012)

SAXENA, S., BAgchi, S., RaO, B.S., NAIK, P.A. \& ChaKerA, J.A.: Single-Shot Terahertz Time Profiling Using Curved Wavefront. IEEE Transactions on Terahertz Science and Technology 8, 528-534 (2018). https://doi.org/10.1109/tthz.2018.2851154

Sharma, D., Singh, D. \& MALIK, H.K.: Shape-Dependent Terahertz Radiation Generation Through Nanoparticles. Plasmonics 15, 177-187 (2020). https://doi.org/10.1007/s11468-019-01017-5.

SHENG, Z.-M., MiMA, K. \& ZHANG, J.: Powerful terahertz emission from laser wake fields excited in inhomogeneous plasmas. Physics of Plasmas 12, 123103 (2005a). https://doi.org/10.1063/1.2136107.

ShEng, Z.-M., Mima, K., Zhang, J. \& SANUKi, H.: Emission of Electromagnetic Pulses from Laser Wakefields through Linear Mode Conversion. Physical Review Letters 94, 095003 (2005b). https://doi.org/10.1103/PhysRevLett.94.095003.

SINGH, D. \& MALIK, H.K.: Terahertz generation by mixing of two super-Gaussian laser beams in collisional plasma. Physics of Plasmas 21, 083105 (2014). https://doi.org/10.1063/1.4891878.

SingH, M. \& SHARMA, R.P.: Generation of THz Radiation by Laser Plasma Interaction. $\begin{array}{lllll}\text { Contributions to Plasma } & \text { Physics 53, 540-548 }\end{array}$ https://doi.org/https://doi.org/10.1002/ctpp.201300006. 
Strickland, D. \& Mourou, G.: Compression of amplified chirped optical pulses. Optics Communications 55, 447-449 (1985). https://doi.org/https://doi.org/10.1016/00304018(85)90151-8.

SzYMAŃSKI, M., Szerling, A. \& Kosiel, K.: Theoretical investigation of metal-metal waveguides for terahertz quantum-cascade lasers. Optical and Quantum Electronics 47, 843-849 (2015). https://doi.org/10.1007/s11082-014-0007-z.

Takano, K., Asai, M., Kato, K., Komiyama, H., Yamaguchi, A., Iyoda, T., Tadokoro, Y., NAKAJIMA, M. \& BAKUnOV, M.I. (2019). Terahertz emission from gold nanorods irradiated by ultrashort laser pulses of different wavelengths. In Sci Rep Ed.^Eds.), Vol. 9, pp. 3280.

VARShNEY, P., GOPAL, K. \& UPADHYAY, A.: Terahertz emission from nonlinear interaction of laser beat wave with nanoparticles. Laser Physics Letters 17, 126002 (2020). https://doi.org/10.1088/1612-202x/abbeea.

Varshney, P., Sajal, V., Singh, K.P., Kumar, R. \& Sharma, N.K.: Tunable and efficient terahertz radiation generation by photomixing of two super Gaussian laser pulses in a corrugated magnetized plasma. Journal of Applied Physics 117, 193303 (2015). https://doi.org/10.1063/1.4921357.

Varshney, P., Upadhayay, A., Madhubabu, K., Sajal, V. \& Chakera, J.A.: Strong terahertz radiation generation by cosh-Gaussian laser beams in axially magnetized collisional plasma under non-relativistic ponderomotive regime. Laser and Particle Beams 36, 236-245 (2018). https://doi.org/Doi: 10.1017/s0263034618000216.

YoshiI, J., LaI, C.H., Katsouleas, T., Joshi, C. \& MoRI, W.B.: Radiation from Cerenkov Wakes in a Magnetized Plasma. Physical Review Letters 79, 4194-4197 (1997). https://doi.org/10.1103/PhysRevLett.79.4194. 